\title{
Coronary Artery Disease: Optimal Lipoprotein(a) for Survival-Lower Is Better? A Large Cohort With 43,647 Patients
}

\section{OPEN ACCESS}

Edited by:

Diego Arroyo,

Fribourg Cantonal

Hospital, Switzerland

Reviewed by:

Gregor Leibundgut,

Cantonal Hospital Baselland

(KSBL), Switzerland

Jinwei Tian,

The Second Affiliated Hospital of Harbin Medical University, China

${ }^{*}$ Correspondence:

Yong Liu

liuyong@gdph.org.cn

tThese authors have contributed equally to this work

Specialty section:

This article was submitted to

Coronary Artery Disease,

a section of the journal

Frontiers in Cardiovascular Medicine

Received: 22 February 2021

Accepted: 09 June 2021

Published: 31 August 2021

Citation:

Liu J, Liu L, Wang B, Chen S, Liu B, Liang J, Huang H, Li Q, Lun Z, Ying $M$, Chen G, Huang Z, Xu D, Yan X, Zhu T, Tadesse GA, Tan N, Chen J and Liu Y

(2021) Coronary Artery Disease:

Optimal Lipoprotein(a) for

Survival-Lower Is Better? A Large Cohort With 43,647 Patients.

Front. Cardiovasc. Med. 8:670859.

doi: 10.3389/fcvm.2021.670859
Jin Liu ${ }^{1 \dagger}$, Liwei Liu ${ }^{1,2 \dagger}$, Bo Wang ${ }^{1 \dagger}$, Shiqun Chen ${ }^{1 \dagger}$, Buyun Liu ${ }^{2}$, Jingjing Liang ${ }^{3}$, Haozhang Huang ${ }^{3}$, Qiang Li $^{1}$, Zhubin Lun ${ }^{4}$, Ming Ying ${ }^{1}$, Guanzhong Chen ${ }^{5}$, Zhidong Huang ${ }^{1}$, Danyuan $\mathrm{Xu}^{1}$, Xiaoming Yan ${ }^{6}$, Tingting $\mathrm{Zhu}^{7}$, Girmaw Abebe Tadesse ${ }^{7}$, Ning Tan ${ }^{1,3,5}$, Jiyan Chen ${ }^{1,3,5}$ and Yong Liu ${ }^{1,3,5 *}$

${ }^{1}$ Department of Cardiology, Guangdong Provincial Key Laboratory of Coronary Heart Disease Prevention, Guangdong Cardiovascular Institute, Guangdong Provincial People's Hospital, Guangdong Academy of Medical Sciences, Guangzhou, China, ${ }^{2}$ Department of Epidemiology, College of Public Health, University of lowa, lowa City, IA, United States, ${ }^{3}$ The Second School of Clinical Medicine, Southern Medical University, Guangzhou, China, ${ }^{4}$ Department of Cardiology, Dongguan TCM Hospital, Dongguan, China, ${ }^{5}$ Guangdong Provincial People's Hospital, School of Medicine, South China University of Technology, Guangzhou, China, ${ }^{6}$ Department of Information Technology, Guangdong Provincial People's Hospital, Guangdong Academy of Medical Sciences, Guangzhou, China, ' Department of Engineering Science, Institute of Biomedical Engineering, University of Oxford, Oxford, United Kingdom

Background: A high level of lipoprotein(a) can lead to a high risk of cardiovascular events or mortality. However, the association of moderately elevated lipoprotein(a) levels $(\geq 15$ $\mathrm{mg} / \mathrm{dL}$ ) with long-term prognosis among patients with coronary artery disease (CAD) is still uncertain. Hence, we aim to systematically analyzed the relevance of baseline plasma lipoprotein(a) levels to long-term mortality in a large cohort of CAD patients.

Methods: We obtained data from 43,647 patients who were diagnosed with CAD and had follow-up information from January 2007 to December 2018. The patients were divided into two groups ( $<15$ and $\geq 15 \mathrm{mg} / \mathrm{dL}$ ). The primary endpoint was long-term all-cause death. Kaplan-Meier curve analysis and Cox proportional hazards models were used to investigate the association between moderately elevated baseline lipoprotein(a) levels ( $\geq 15 \mathrm{mg} / \mathrm{dL}$ ) and long-term all-cause mortality.

Results: During a median follow-up of 5.04 years, 3,941 (18.1\%) patients died. We observed a linear association between lipoprotein(a) levels and long-term all-cause mortality. Compared with lipoprotein(a) concentrations $<15 \mathrm{mg} / \mathrm{dL}$, lipoprotein(a) $\geq 15$ $\mathrm{mg} / \mathrm{dL}$ was associated with a significantly higher risk of all-cause mortality [adjusted hazard ratio (aHR) 1.10, 95\%Cl: 1.04-1.16, P-values $=0.001)$. Similar results were found for the subgroup analysis of non-acute myocardial infarction, non-percutaneous coronary intervention, chronic heart failure, diabetes mellitus, or non-chronic kidney diseases. 
Conclusion: Moderately elevated baseline plasma lipoprotein(a) levels ( $\geq 15 \mathrm{mg} / \mathrm{dL}$ ) are significantly associated with higher all-cause mortality in patients with CAD. Our finding provides a rationale for testing the lipoprotein(a)-reducing hypothesis with lower targets (even $<15 \mathrm{mg} / \mathrm{dL}$ ) in CAD outcome trials.

Keywords: coronary artery disease, baseline lipoprotein(a), long-term all-cause mortality, percutaneous coronary intervention, coronary angiography

\section{INTRODUCTION}

In the field of epidemiology and genetics, numerous studies have found that plasma lipoprotein(a) remains a residual risk factor for atherosclerotic cardiovascular disease (ASCVD) (1-4). Elevated plasma lipoprotein(a) levels are consequently associated with an increased risk of adverse clinical events because of the atherogenic and thrombogenic properties of lipoprotein(a) (4-7).

The 2019 ESC/EAS dyslipidemia guidelines suggested that lipoprotein(a) should be measured in people with high ASCVD risk, and elevated lipoprotein(a) ( $\geq 50 \mathrm{mg} / \mathrm{dL}$ ) would be regarded as a significant risk factor of cardiovascular disease; however, the dose effect of lipoprotein(a) with long-term prognosis among ASCVD patients is uncertain (8). Several studies found that a baseline plasma lipoprotein(a) $>30 \mathrm{mg} / \mathrm{dL}$ would increase the risk of cardiovascular events or all-cause mortality (7, 9-11). A Vera et al. indicated that baseline lipoprotein(a) levels were not significantly associated with long-term mortality among ACS patients (12). As a whole, the effect of moderately elevated lipoprotein(a) levels $(\geq 15 \mathrm{mg} / \mathrm{dL})$ for long-term mortality among patients with CAD was still uncertain. The definitive lipoprotein(a) levels may provide a more accurate risk stratification for indicating a high risk of CAD patients and may prompt an important reference for the subsequent improvement of long-term prognosis among CAD patients via a lipoprotein(a)lowering treatment.

Therefore, we systematically analyzed the relevance of moderately elevated lipoprotein(a) levels $(\geq 15 \mathrm{mg} / \mathrm{dL}$ ) to longterm mortality in a large cohort of CAD patients.

\section{METHODS}

\section{Study Design and Participants}

The study was based on a total of 88,938 patients as confirmed by coronary angiography (CAG) from January 2007 to December 2018 at Guangdong Provincial People's Hospital in China (Clinical Trials.gov NCT04407936), and the retrospective observational study included 59,667 patients with a final diagnosis of CAD according to the 10th Revision Codes of the International Classification of Diseases (ICD-10; I20.xxI25.xx, I50.00001 and I91.40001, Supplementary Table 2). After excluding 8,723 patients who lacked a lipoprotein(a) examination, a total of 50,944 patients were enrolled in the analyses subsequently, with lack of follow-up information of CAD mortality $(n=7,297)$. The final analysis included 43,647 CAD patients who underwent the first CAG during the study period. The study protocol was approved by the Guangdong
Provincial People's Hospital Ethics Committee, and the study was performed according to the Declaration of Helsinki.

Baseline data were extracted from the electronic Clinical Management System of the Guangdong Provincial People's Hospital. The baseline information mainly included demographic characteristics, coexisting conditions, laboratory examinations, and medications at discharge. CAG or percutaneous coronary intervention (PCI) was performed following standard clinical practice guidelines (13-15).

\section{Clinical Definition}

Chronic kidney disease (CKD) was defined as an estimated glomerular filtration rate $(\mathrm{eGFR})<60 \mathrm{~mL} / \mathrm{min} / 1.73 \mathrm{~m}^{2}$, and eGFR was calculated using the Modification of Diet in Renal Disease equation (16). Congestive heart failure (CHF) was defined as New York Heart Association class $>2$ or Killip class $>1$. Diabetes mellitus (DM) and hypertension were defined using ICD-10 codes (Supplementary Table 2 ).

\section{Study Outcome}

The primary endpoint was long-term all-cause death, and incident events were defined as the first event occurring between the date of enrollment and the end of follow-up on December 31, 2018. This information was monitored and recorded by research assistants and trained nurses through outpatient interviews and telephones. The concentration of lipoprotein(a) was measured for the first time in the hospital via immunoturbidimetry on a chemistry analyzer (AU5800 Analyzer, Beckman Coulter, Brea, California).

\section{Statistical Analysis}

The statistical analysis for this study was performed from January 1, 2007 to December 31, 2018. The patients were divided into normal group $(<15 \mathrm{mg} / \mathrm{dL})$ and moderately elevated group $(\geq 15$ $\mathrm{mg} / \mathrm{dL}$ ) by lipoprotein(a) concentration. Continuous variables were tested for normality with visual inspection (histograms and normal Q-Q plot) and expressed as mean (standard deviation, $\mathrm{SD})$. The descriptive statistics for continuous variables with abnormal distribution and categorical variables are reported as median (interquartile range, IQR) and numbers (percentages), respectively. Student's $t$-test and Wilcoxon rank-sum test were used to analyze differences across groups as appropriate. Pearson chi-square tests were used to analyze the categorical data.

Prognosis analysis was performed using Kaplan-Meier methods, and survival curves were used for the probability of the remaining outcome-free in the two groups. We used the log-rank test to compare the survival differences among the two groups. 
TABLE 1 | Baseline characteristics of the patients.

\begin{tabular}{|c|c|c|c|c|}
\hline Characteristic* & $\begin{array}{c}\text { Overall } \\
(n=43,647)\end{array}$ & $\begin{array}{c}\mathrm{LPa}<15 \mathrm{mg} / \mathrm{dL} \\
(n=19,945)\end{array}$ & $\begin{array}{c}\mathrm{LPa} \geq 15 \mathrm{mg} / \mathrm{dL} \\
(n=23,702)\end{array}$ & $P$-value \\
\hline \multicolumn{5}{|l|}{ Demographic characteristics } \\
\hline Age, years, mean (SD) & $63.1(10.7)$ & $62.8(10.8)$ & $63.3(10.6)$ & $<0.001$ \\
\hline Female, $n(\%)$ & 10,449 (23.9) & 4,649 (23.3) & $5,800(24.5)$ & 0.005 \\
\hline \multicolumn{5}{|l|}{ Medical history } \\
\hline AMl, $n(\%)$ & $8,584(19.7)$ & $3,321(16.7)$ & $5,263(22.2)$ & $<0.001$ \\
\hline $\mathrm{CHF}, n(\%)$ & $4,146(9.5)$ & $1,611(8.1)$ & $2,535(10.7)$ & $<0.001$ \\
\hline Hypertension, $n$ (\%) & $24,619(56.5)$ & $11,436(57.4)$ & $13,183(55.7)$ & $<0.001$ \\
\hline $\mathrm{DM}, n(\%)$ & $12,008(27.5)$ & $5,703(28.6)$ & $6,305(26.6)$ & $<0.001$ \\
\hline $\mathrm{PCl}, n(\%)$ & $31,688(72.6)$ & $13,843(69.4)$ & $17,845(75.3)$ & $<0.001$ \\
\hline CKD, $n(\%)$ & $7,651(17.5)$ & $3,011(15.1)$ & 4,640 (19.6) & $<0.001$ \\
\hline \multicolumn{5}{|l|}{ Laboratory tests } \\
\hline Lipoprotein(a), mg/dL, mean (SD) & $29.61(33.18)$ & $8.41(3.50)$ & $47.44(36.34)$ & $<0.001$ \\
\hline WBC, $10^{9} / \mathrm{L}$, mean (SD) & $7.98(2.73)$ & $7.90(2.74)$ & $8.05(2.72)$ & $<0.001$ \\
\hline HGB, g/L, mean (SD) & $133.28(16.81)$ & $134.83(16.11)$ & $131.98(17.27)$ & $<0.001$ \\
\hline $\mathrm{CHOL}, \mathrm{mmol} / \mathrm{L}$, mean (SD) & $4.54(1.21)$ & $4.38(1.15)$ & $4.68(1.24)$ & $<0.001$ \\
\hline TRIG, mmol/L, mean (SD) & $1.66(1.22)$ & $1.80(1.47)$ & $1.54(0.94)$ & $<0.001$ \\
\hline APOA, g/L, mean (SD) & $1.54(0.94)$ & $1.11(0.26)$ & $1.09(0.26)$ & $<0.001$ \\
\hline APOB, g/L, mean (SD) & $0.86(0.24)$ & $0.82(0.23)$ & $0.89(0.25)$ & $<0.001$ \\
\hline LDLC, mmol/L, mean (SD) & $2.81(0.97)$ & $2.64(0.91)$ & $2.95(1.00)$ & $<0.001$ \\
\hline HDLC, mmol/L, mean (SD) & $1.00(0.26)$ & $0.99(0.26)$ & $1.01(0.26)$ & $<0.001$ \\
\hline HbA1c, \%, mean (SD) & $6.55(1.41)$ & $6.54(1.38)$ & $6.56(1.44)$ & 0.231 \\
\hline URIC, $\mu \mathrm{mol} / \mathrm{L}$, mean (SD) & $396.45(112.37)$ & $397.36(109.72)$ & 395.68 (114.56) & 0.159 \\
\hline eGFR, mL/min/1.73 m², mean (SD) & $77.51(24.80)$ & $79.49(24.12)$ & $75.89(25.22)$ & $<0.001$ \\
\hline \multicolumn{5}{|l|}{ Medications } \\
\hline ACEI/ARB, $n(\%)$ & $21,180(49.3)$ & $9,446(48.1)$ & $11,734(50.4)$ & $<0.001$ \\
\hline Beta-blockers, $n(\%)$ & $34,774(81.0)$ & $15,760(80.3)$ & $19,014(81.6)$ & $<0.001$ \\
\hline Statins, $n(\%)$ & 40,715 (94.9) & $18,565(94.6)$ & $22,150(95.1)$ & 0.012 \\
\hline
\end{tabular}

LPa, lipoprotein(a); AMI, acute myocardial infarction; CHF, congestive heart failure; DM, diabetes mellitus; PCI, percutaneous coronary intervention; CKD, chronic kidney disease; WBC, white blood cell; HGB, hemoglobin; $C H O$, serum total cholesterol; TG, triglycerides; $A P O A$, apolipoprotein A; $A P O B$, apolipoprotein B; LDL-C, low-density lipoprotein cholesterol; HDL-C, high-density lipoprotein cholesterol; eGFR, estimated glomerular filtration rate; ACEI/ARB, angiotensin-converting enzyme inhibitor/angiotensin receptor blocker.

Cox proportional hazard regression models and restricted cubic splines were used to investigate the associations of baseline lipoprotein(a) levels with long-term all-cause mortality. Hazard ratio and 95\% confidence interval (CI) were reported. Model 1 was univariate Cox analysis. Model 2 was to adjust age (as a continuous variable) and gender. Model 3 included age, gender, and four lipid continuous variables (total cholesterol, triglyceride, apolipoprotein A, and low-density lipoprotein cholesterol), and model 4 included the variables which were significant at $P<0.05$ according to univariate Cox proportional hazard regression and those associated with mortality according to clinical experience (including models 2 and 3 variables, history of present illness information, and drug information). Furthermore, we additionally adjusted apolipoprotein B, fibrinogen, and hypersensitive C-reactive protein, respectively, based on model 4 . Finally, we defined the results of model 4 as the primary results. When data were missing, we used the last available observation.

We used model 3 to perform a sensitivity analysis in five different subgroups [PCI, acute myocardial infarction
(AMI), CKD, DM, and CHF]. All data analyses were performed using R (version 3.6.3; R Core Team, Vienna, Austria). $P$-values $<0.05$ were considered to represent statistical significance.

\section{RESULT}

\section{Clinical Characteristics}

From January 2007 to December 2018, a total of 43,647 CAD patients were enrolled in the study. The mean age was $63.1 \pm 10.7$ years, and 33,198 (76.1\%) were male. The patients were divided into two groups according to the median level of lipoprotein(a) concentration: lower lipoprotein(a) group [lipoprotein(a) $<15 \mathrm{mg} / \mathrm{dL}, n=19,945]$ and higher lipoprotein(a) group [lipoprotein(a) $\geq 15 \mathrm{mg} / \mathrm{dL}, n=23,702$ ]. Among the subgroup analyses, 31,688 (72.6\%) patients underwent PCI treatment, and 9,541 (22.9\%) patients were identified as CKD, 12,008 (27.5\%) patients were complicated with DM, and 4,160 (9.5\%) had CHF (Table 1). 


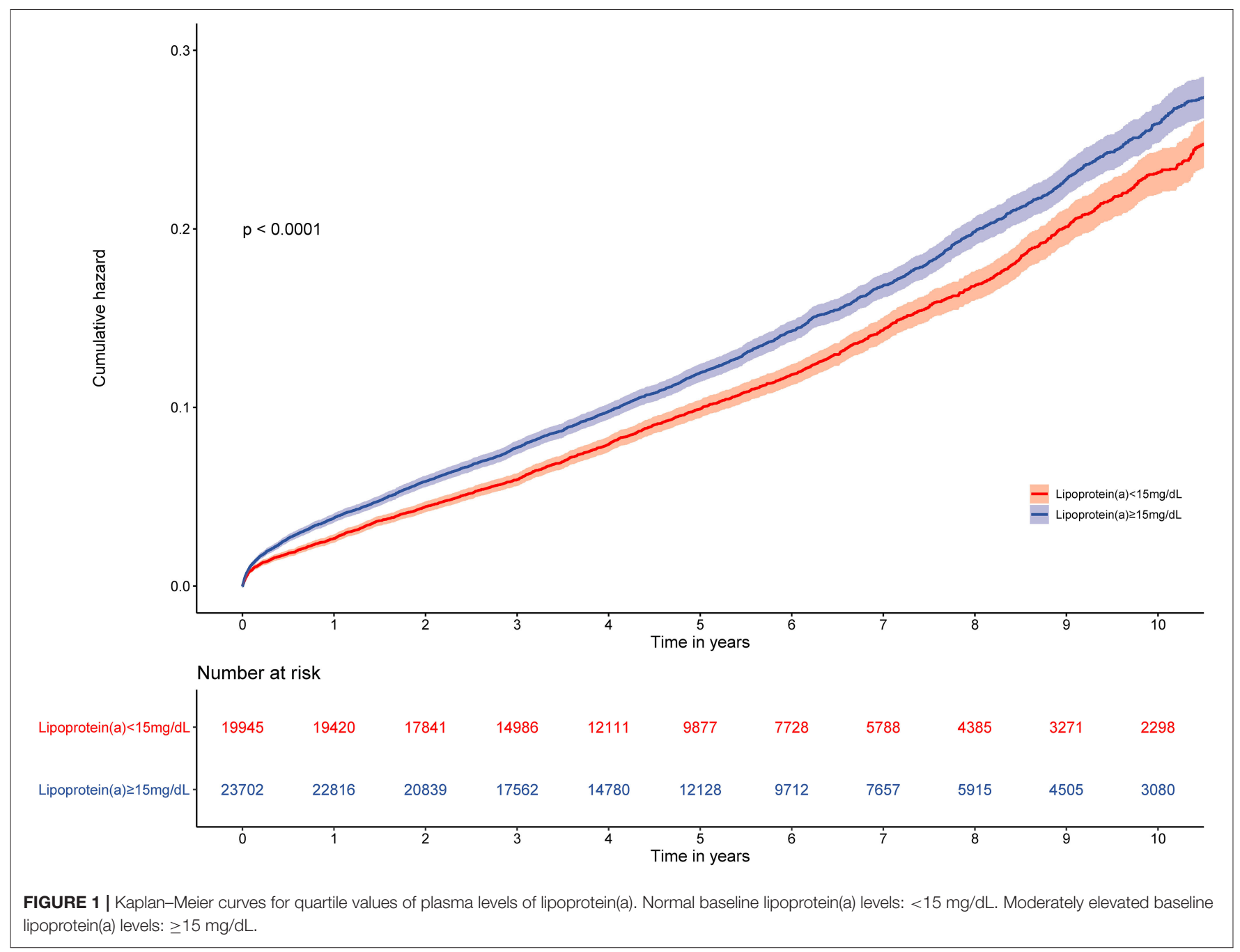

\section{Primary Outcomes}

The median level of lipoprotein was $15.0 \mathrm{mg} / \mathrm{dL}$. In total, there were 3,941 (18.1\%) deaths during a median followup of 5.0 years (IQR, 3.0-7.8; Table 1), and patients with lipoprotein(a) $\geq 15 \mathrm{mg} / \mathrm{dL}$ has a higher mortality rate than those with lipoprotein(a) $<15 \mathrm{mg} / \mathrm{dL}$ [3,391 (14.3\%) vs. 2,381 (11.9\%), $P<0.001$, respectively]. The level of lipoprotein(a) did not significantly differ with age but was higher in females (Supplementary Figure 2).

The prevalence of total mortality in the lower and higher lipoprotein(a) groups according to the median of lipoprotein(a) levels were 6.4 and 9.0\%, respectively. As determined by Kaplan-Meier analysis, the elevated lipoprotein(a) levels $(\geq 15$ $\mathrm{mg} / \mathrm{dL}$ ) led to worse long-term prognosis (log-rank $P<0.001$, Figure 1). The univariate regression analysis showed that 25 variables (including age, sex, etc.) were significantly associated with the primary endpoint (Supplementary Table 1). In the analyses of univariate and multivariate models, we observed an approximate linear association between lipoprotein(a) levels and long-term all-cause mortality (Figure 2). According to the results of the multivariable-adjusted models, lipoprotein(a) levels $\geq 15 \mathrm{mg} / \mathrm{dL}$ increased the risk of all-cause death by $10 \%$ compared with lipoprotein(a) levels $<15 \mathrm{mg} / \mathrm{dL}$ (aHR, 1.10; 95\%CI, 1.04-1.16) (Figure 3).

The subgroup analyses also suggested that the results did not change substantially among patients who underwent PCI (aHR, 1.95; 95\%CI, 0.98-1.12), AMI (aHR, 1.02; 95\%CI, 0.90-1.15), non-DM (aHR, 1.06; 95\%CI, 0.99-1.14), and CHF (aHR, 1.10, 95\%CI, 1.04-1.17) (Figure 4).

\section{DISCUSSION}

To our knowledge, the present study provides the first systematical evaluation of the association of moderately elevated baseline plasma lipoprotein(a) and all-cause mortality in a large cohort of CAD patients. Our data showed that lipoprotein(a) levels $\geq 15 \mathrm{mg} / \mathrm{dL}$ were strongly associated with all-cause death in patients with CAD. Similar results were observed in the subgroup analysis [non-PCI, CKD (yes or no), non-AMI or DM and non-CHF).

An earlier study indicated that lipoprotein(a) is a residual risk factor in patients who achieved target lipid levels by the 


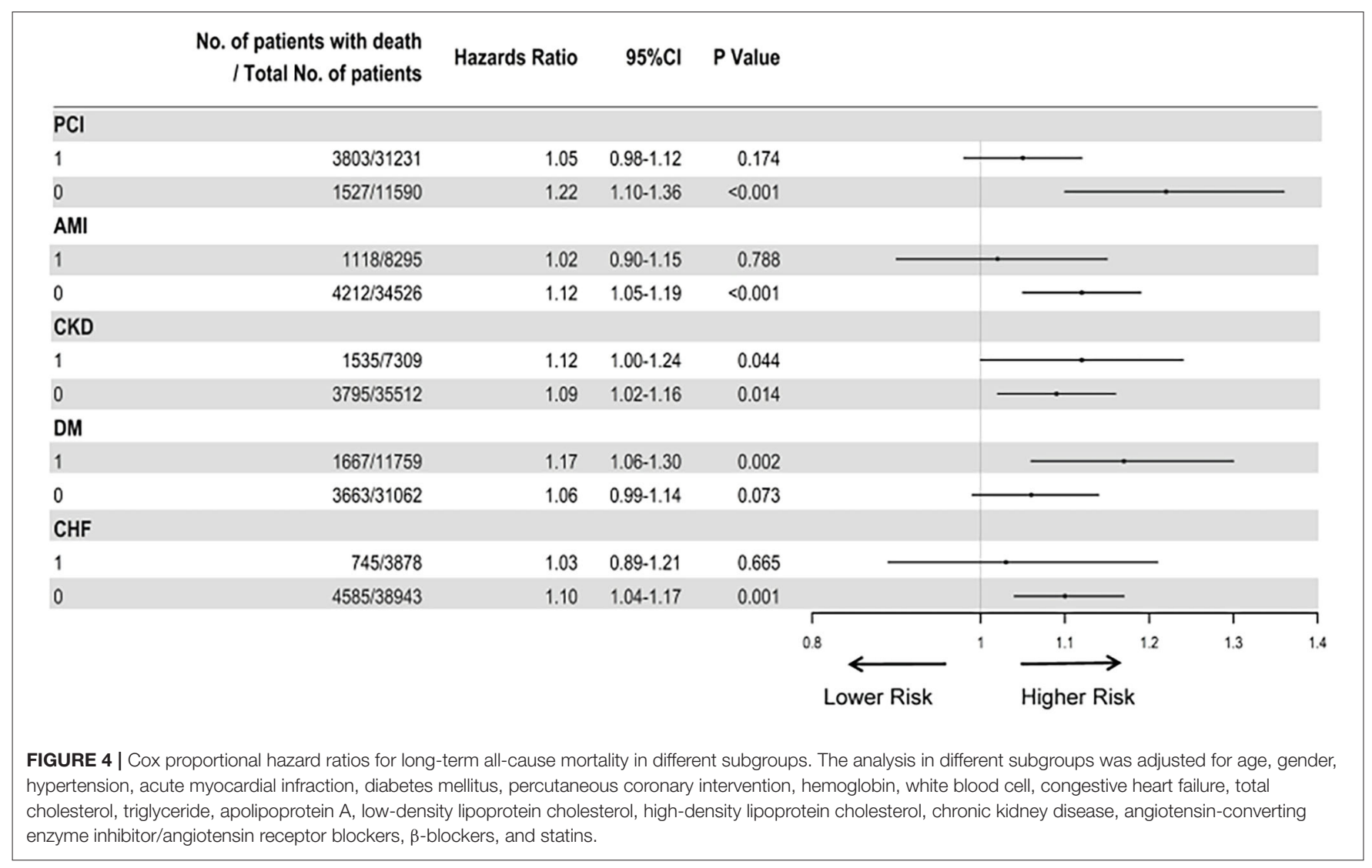

high atherosclerotic cardiovascular disease risk, and when the lipoprotein(a) concentration is above $50 \mathrm{mg} / \mathrm{dL}$, it would be regarded as a significant risk factor of prognosis (8). Because previous studies mainly included the general population or limited samples of CAD patients, the associations between specific elevated plasma levels of lipoprotein(a) and long-term all-cause mortality in CAD are limited (5, 7, 19-21). In addition, there is no definitive clinical recognition of the cutoff value of lipoprotein(a) associated with long-term all-cause mortality. Therefore, we included large samples (more than 40,000) of CAD patients to further identify the relationship between lipoprotein(a) levels and long-term prognosis. After adjusting for demographic characteristics, medical history, laboratory tests, and medication variables $(8,22)$, the moderately elevated baseline lipoprotein(a) levels ( $\geq 15 \mathrm{mg} / \mathrm{dL}$ ) remained significantly associated with an unfavorable prognosis. Furthermore, there was good statistical evidence for a similar result in different subgroups [non-PCI, CKD (yes or no), non-AMI or DM and non-CHF].

Our study found that lipoprotein(a) is an independent risk factor for all-cause mortality, which should be tested among CAD patients. Furthermore, CAD patients with lipoprotein(a) level $\geq 15 \mathrm{mg} / \mathrm{dL}$ had a higher risk of all-cause mortality, and they might need a lipoprotein(a)-lowering treatment. Many studies have revealed that PCSK9i can decrease the lipoprotein(a) concentration, which may considered for practice in CAD patients with moderately elevated lipoprotein(a) $(23,24)$.
Lipoprotein(a) is a plasma lipoprotein composed of apolipoprotein(a) covalently bound to apolipoprotein B100, a low-density lipoprotein-like particle. Previous studies have shown a significant relationship between high lipoprotein(a) levels and cardiovascular disease $(4,25)$. Patients with high levels of lipoprotein(a) tend to have more severe cardiovascular conditions, which may lead to an increased risk of all-cause death. It is generally believed that lipoprotein(a) participates in the pathophysiological process of coronary artery disease by promoting atherosclerosis or inflammation (26). In addition, lipoprotein(a) may promote the thrombotic state through a variety of mechanisms, including inhibition of the fibrinolytic system and enhancement of tissue factor-mediated pathways $(27,28)$. Although high lipoprotein(a) levels may protect against bleeding events, thrombosis plays a more crucial role in the poor prognosis among patients with existing CAD.

\section{Strength and Limitation}

This study examined for the first time the association of baseline plasma lipoprotein(a) and all-cause mortality in a large cohort of CAD patients. The abundant data extracted from medical records allowed us to control for a variety of confounders in the analyses. There are several limitations to this study. First, information about cause-specific death was not available in this study, which restricted our ability to examine the association of lipoprotein(a) with cause-specific death, such as CVD mortality. Second, only in-hospital baseline lipoprotein(a) was contained 
in our study. Therefore, we could not know the status of lipoprotein(a) after discharge and the effects of its change. However, we are further collecting the follow-up lipoprotein(a) information after discharge. Third, although we have adjusted for many confounders in the analyses, residual confounding due to unmeasured factors was still available (such as socioeconomic factors, body mass index, and lifestyle factors).

\section{CONCLUSION}

Moderately elevated plasma lipoprotein(a) levels were significantly associated with an increased risk of all-cause mortality among patients with confirmed CAD, and moderately elevated baseline plasma lipoprotein(a) ( $\geq 15 \mathrm{mg} / \mathrm{dL})$ could identify the risk of long-term all-cause mortality in patients with CAD. Our finding highlighted the significance of plasma lipoprotein(a) as an independent and long-term prognostic indicator for CAD patients. Future studies are needed to confirm our findings.

\section{DATA AVAILABILITY STATEMENT}

The raw data supporting the conclusions of this article will be made available by the authors, without undue reservation.

\section{ETHICS STATEMENT}

Ethical review and approval was not required for the study on human participants in accordance with the local legislation and institutional requirements. The patients/participants provided their written informed consent to participate in this study.

\section{AUTHOR CONTRIBUTIONS}

YL, JLiu, NT, and JC conceptualized the research idea and study design. JLiu, ZL, LL, JLia, SC, BW, QL, HH, and ZH contributed to data acquisition. JLiu and YL contributed to data analysis/interpretation. DX performed the statistical analysis.

\section{REFERENCES}

1. Nordestgaard BG, Chapman MJ, Ray K, Boren J, Andreotti F, Watts GF, et al. Lipoprotein(a) as a cardiovascular risk factor: current status. Eur Heart J. (2010) 31:2844-53. doi: 10.1093/eurheartj/ ehq386

2. Kronenberg F, Utermann G. Lipoprotein(a): resurrected by genetics. J Intern Med. (2013) 273:6-30. doi: 10.1111/j.1365-2796.2012.02592.x

3. Tsimikas S. A test in context: Lipoprotein(a): diagnosis, prognosis, controversies, emerging therapies. J Am Coll Cardiol. (2017) 69:692711. doi: 10.1016/j.jacc.2016.11.042

4. Wu MF, Xu KZ, Guo YG, Yu J, Wu Y, Lin LM. Lipoprotein(a) and atherosclerotic cardiovascular disease: current understanding and future perspectives. Cardiovasc Drugs Ther. (2019) 33:73948. doi: 10.1007/s10557-019-06906-9

5. Cressman MD, Heyka RJ, Paganini EP, O’Neil J, Skibinski CI, Hoff HF. Lipoprotein(a) is an independent risk factor for cardiovascular disease in hemodialysis patients. Circulation. (1992) 86:475-82. doi: 10.1161/01.CIR.86.2.475
YL, JC, and NT took charge of supervision and mentorship. $\mathrm{XY}, \mathrm{GAT}$, and BL provided writing guidance. Each author contributed important intellectual content during manuscript drafting or revision and accepts accountability for the overall work by ensuring that questions pertaining to the accuracy or integrity of any portion of the work are appropriately investigated and resolved.

\section{FUNDING}

This study was supported by grants from Beijing Lisheng Cardiovascular Health Foundation, Guangdong Provincial People's Hospital Foundation (LHJJ20141751), Natural Science Foundation of Guangdong Province General Project (2020A1515010940) and National Science Foundation of China (81670339 and 81970311). The funders had no role in the study design, data collection and analysis, decision to publish, or preparation of the manuscript. This work was not funded by any industry sponsors. The funding sources had no role in the design and conduct of the study; collection, management, analysis, and interpretation of the data; preparation, review, or approval of the manuscript; or decision to submit the manuscript for publication.

\section{ACKNOWLEDGMENTS}

Special thanks go to Professor Ying Xian (Duke Clinical Research Institute, Durham, NC, 27705, USA) for helping with the study design. We thank BL (College of Public Health, University of Iowa) for her precious comments. TZ was supported by the RAEng Engineering for Development Research Fellowship.

\section{SUPPLEMENTARY MATERIAL}

The Supplementary Material for this article can be found online at: https://www.frontiersin.org/articles/10.3389/fcvm. 2021.670859/full\#supplementary-material

6. Dai W, Long J, Cheng Y, Chen Y, Zhao S. Elevated plasma lipoprotein(a) levels were associated with increased risk of cardiovascular events in Chinese patients with stable coronary artery disease. Sci Rep. (2018) 8:7726. doi: 10.1038/s41598-018-25835-5

7. Jin JL, Cao YX, Zhang HW, Sun D, Hua Q, Li YF, et al. Lipoprotein(a) and cardiovascular outcomes in patients with coronary artery disease and prediabetes or diabetes. Diabetes Care. (2019) 42:1312-8. doi: 10.2337/dc19-0274

8. Mach F, Baigent C, Catapano AL, Koskinas KC, Casula M, Badimon L, et al. 2019 ESC/EAS Guidelines for the management of dyslipidaemias: lipid modification to reduce cardiovascular risk. Eur Heart J. (2020) 41:11188. doi: 10.15829/1560-4071-2020-3826

9. Glader CA, Birgander LS, Stenlund H, Dahlén GH. Is lipoprotein(a) a predictor for survival in patients with established coronary artery disease? Results from a prospective patient cohort study in northern Sweden. J Intern Med. (2002) 252:27-35. doi: 10.1046/j.1365-2796.2002.00997.x

10. Wang Z, Zhai X, Xue M, Cheng W, Hu H. Prognostic value of lipoprotein (a) level in patients with coronary artery disease: a meta-analysis. Lipids Health Dis. (2019) 18:150. doi: 10.1186/s12944-019-1092-6 
11. Park SH, Rha SW, Choi BG, Park JY, Jeon U, Seo HS, et al. Impact of high lipoprotein(a) levels on in-stent restenosis and long-term clinical outcomes of angina pectoris patients undergoing percutaneous coronary intervention with drug-eluting stents in Asian population. Clin Exp Pharmacol Physiol. (2015) 42:588-95. doi: 10.1111/1440-1681.12396

12. Bittner V, Szarek M, Aylward P, Bhatt D, Diaz R, Edelberg J, et al. Effect of alirocumab on lipoprotein(a) and cardiovascular risk after acute coronary syndrome. J Am Coll Cardiol. (2020) 75:133-44. doi: 10.1016/j.jacc.2019.10.057

13. Kushner FG, Hand M, Smith SC, Jr., King SB, III, Anderson JL, Antman EM, et al. 2009 focused updates: ACC/AHA guidelines for the management of patients with ST-elevation myocardial infarction (updating the 2004 guideline and 2007 focused update) and ACC/AHA/SCAI guidelines on percutaneous coronary intervention (updating the 2005 guideline and 2007 focused update) a report of the American College of Cardiology Foundation/American Heart Association Task Force on Practice Guidelines. J Am Coll Cardiol. (2009) 54:2205-41. doi: 10.1161/CIRCULATIONAHA.109.192663

14. Jneid H, Anderson JL, Wright RS, Adams CD, Bridges CR, Casey DE, et al. 2012 ACCF/AHA focused update of the guideline for the management of patients with unstable angina/non-ST-elevation myocardial infarction (updating the 2007 guideline and replacing the 2011 focused update): a report of the American College of Cardiology Foundation/American Heart Association Task Force on Practice Guidelines. J Am Coll Cardiol. (2012) 60:645-81. doi: 10.1161/CIR.0b013e318256f1e0

15. Levine GN, Bates ER, Blankenship JC, Bailey SR, Bittl JA, Cercek B, et al. 2015 ACC/AHA/SCAI focused update on primary percutaneous coronary intervention for patients with ST-elevation myocardial infarction: an update of the $2011 \mathrm{ACCF} / \mathrm{AHA} / \mathrm{SCAI}$ guideline for percutaneous coronary intervention and the 2013 ACCF/AHA guideline for the management of ST-elevation myocardial infarction. J Am Coll Cardiol. (2016) 67:123550. doi: 10.1016/j.jacc.2015.10.005

16. Aguiar-Souto P, Ferrante G, Del Furia F, Barlis P, Khurana R, Di Mario C. Frequency and predictors of contrast-induced nephropathy after angioplasty for chronic total occlusions. Int J Cardiol. (2010) 139:6874. doi: 10.1016/j.ijcard.2008.10.006

17. Konishi H, Miyauchi K, Kasai T, Tsuboi S, Ogita M, Naito R, et al. Impact of lipoprotein(a) as residual risk on long-term outcomes in patients after percutaneous coronary intervention. Am J Cardiol. (2015) 115:15760. doi: 10.1016/j.amjcard.2014.10.015

18. Shim YS, Baek JW, Kang MJ, Oh YJ, Yang S, Hwang IT. Reference values for the triglyceride to high-density lipoprotein cholesterol ratio and non-high-density lipoprotein cholesterol in korean children and adolescents: the Korean National Health and Nutrition Examination Surveys 2007-2013. J Atheroscler Thromb. (2016) 23:1334-44. doi: 10.5551/jat. 35634

19. Langsted A, Kamstrup PR, Nordestgaard BG. High lipoprotein(a) and high risk of mortality. Eur Heart J. (2019) 40:276070. doi: 10.1093/eurheartj/ehy902

20. Kollerits B, Drechsler C, Krane V, Lamina C, März W, Dieplinger $\mathrm{H}$, et al. Lipoprotein(a) concentrations, apolipoprotein(a) isoforms and clinical endpoints in haemodialysis patients with type 2 diabetes mellitus: results from the 4D Study. Nephrol Dial Transplant. (2016) 31:19018. doi: $10.1093 / \mathrm{ndt} / \mathrm{gfv} 428$
21. Feng Z, Li HL, Bei WJ, Guo XS, Wang K, Yi SX, et al. Association of lipoprotein(a) with long-term mortality following coronary angiography or percutaneous coronary intervention. Clin Cardiol. (2017) 40:6748. doi: $10.1002 /$ clc. 22712

22. Baigent C, Blackwell L, Emberson J, Holland LE, Reith C, Bhala N, et al. Efficacy and safety of more intensive lowering of LDL cholesterol: a metaanalysis of data from 170,000 participants in 26 randomised trials. Lancet (London, England). (2010) 376:1670-81. doi: 10.1016/S0140-6736(10)61350-5

23. Raal FJ, Giugliano RP, Sabatine MS, Koren MJ, Langslet G, Bays H, et al. Reduction in lipoprotein(a) with PCSK9 monoclonal antibody evolocumab (AMG 145): a pooled analysis of more than 1,300 patients in 4 phase II trials. J Am Coll Cardiol. (2014) 63:1278-88. doi: 10.1016/j.jacc.2014.01.006

24. Schwartz GG, Steg PG, Szarek M, Bittner VA, Diaz R, Goodman SG, et al. Peripheral artery disease and venous thromboembolic events after acute coronary syndrome: role of lipoprotein(a) and modification by alirocumab: prespecified analysis of the ODYSSEY OUTCOMES randomized clinical trial. Circulation. (2020) 141:1608-17. doi: 10.1161/CIRCULATIONAHA.120.046524

25. Folch J, Lees M, Sloane Stanley GH. A simple method for the isolation and purification of total lipides from animal tissues. J Biol Chem. (1957) 226:497-509. doi: 10.1016/S0021-9258(18)64849-5

26. van der Valk FM, Bekkering S, Kroon J, Yeang C, Van den Bossche J, van Buul JD, et al. Oxidized phospholipids on lipoprotein(a) elicit arterial wall inflammation and an inflammatory monocyte response in humans. Circulation. (2016) 134:61124. doi: 10.1161/CIRCULATIONAHA.116.020838

27. Deb A, Caplice NM Lipoprotein(a): new insights into mechanisms of atherogenesis and thrombosis. Clin Cardiol. (2004) 27:25864. doi: 10.1002/clc.4960270503

28. Caplice NM, Panetta C, Peterson TE, Kleppe LS, Mueske CS, Kostner GM, et al. Lipoprotein (a) binds and inactivates tissue factor pathway inhibitor: a novel link between lipoproteins and thrombosis. Blood. (2001) 98:29807. doi: 10.1182/blood.V98.10.2980

Conflict of Interest: The authors declare that the research was conducted in the absence of any commercial or financial relationships that could be construed as a potential conflict of interest.

Publisher's Note: All claims expressed in this article are solely those of the authors and do not necessarily represent those of their affiliated organizations, or those of the publisher, the editors and the reviewers. Any product that may be evaluated in this article, or claim that may be made by its manufacturer, is not guaranteed or endorsed by the publisher.

Copyright (C) 2021 Liu, Liu, Wang, Chen, Liu, Liang, Huang, Li, Lun, Ying, Chen, Huang, Xu, Yan, Zhu, Tadesse, Tan, Chen and Liu. This is an open-access article distributed under the terms of the Creative Commons Attribution License (CC BY). The use, distribution or reproduction in other forums is permitted, provided the original author(s) and the copyright owner(s) are credited and that the original publication in this journal is cited, in accordance with accepted academic practice. No use, distribution or reproduction is permitted which does not comply with these terms. 